\title{
MIXED NORM INEQUALITIES FOR SOME DIRECTIONAL MAXIMAL OPERATORS
}

\section{DAH-CHIN LUOR}

\author{
(Received 15 November 2011)
}

\begin{abstract}
Mixed norm inequalities for directional operators are closely related to the boundedness problems of several important operators in harmonic analysis. In this paper we prove weighted inequalities for some one-dimensional one-sided maximal functions. Then by applying these results, we establish mixed norm inequalities for directional maximal operators which are defined from these one-dimensional maximal functions. We also estimate the constants in these inequalities.
\end{abstract}

2010 Mathematics subject classification: primary 42B25; secondary 26D10, 47G10.

Keywords and phrases: weighted inequalities, mixed norm inequalities, directional maximal operators, maximal functions.

\section{Introduction}

Let $\Sigma^{n-1}$ be the unit sphere in $\mathbb{R}^{n}$ and $\Omega$ be a given function over $\mathbb{R}^{n} \times \Sigma^{n-1}$. In [2] Calderón and Zygmund considered homogeneous singular integrals with variable kernel defined by

$$
T_{\Omega} f(x)=\text { p.v. } \int_{\mathbb{R}^{n}} \Omega\left(x, y^{\prime}\right)|y|^{-n} f(x-y) d y,
$$

where $y^{\prime}=y /|y|$. If $\Omega$ is odd in its second variable, then $T_{\Omega}$ can be represented as

$$
T_{\Omega} f(x)=\frac{1}{2} \int_{\Sigma^{n-1}} \Omega(x, \theta) H_{\theta} f(x) d \theta .
$$

Here $H_{\theta}$ is the directional Hilbert transform defined by

$$
H_{\theta} f(x)=\text { p.v. } \int_{\mathbb{R}} f(x-t \theta) t^{-1} d t, \quad x \in \mathbb{R}^{n} .
$$

The boundedness of $T_{\Omega}$ on $L^{p}\left(\mathbb{R}^{n}\right), 1<p<\infty$, can be obtained if $\left(\int_{\Sigma^{n-1}} \Omega(x, \theta)^{r^{*}} d \theta\right)^{1 / r^{*}}$ is bounded as a function of $x$, where $1 \leq r \leq \infty$ and $1 / r+1 / r^{*}=1$, and if the mixed

This work is supported by the I-Shou University of the Republic of China under grant ISU 99-02-25.

(C) 2012 Australian Mathematical Publishing Association Inc. 0004-9727/2012 \$16.00 
norm inequality

$$
\left(\int_{\mathbb{R}^{n}}\left(\int_{\Sigma^{n-1}}\left|S_{\theta} f(x)\right|^{r} d \theta\right)^{q / r} d x\right)^{1 / q} \leq C\left(\int_{\mathbb{R}^{n}}|f(x)|^{p} d x\right)^{1 / p}
$$

holds for $S_{\theta}=H_{\theta}$ and $q=p$; see [2,3]. Here we let $S_{\theta}$ to be a directional operator defined from some one-dimensional operator. Consider the Riesz potentials

$$
I_{\alpha, \Omega} f(x)=\int_{\mathbb{R}^{n}} \Omega\left(x, y^{\prime}\right)|y|^{\alpha-n} f(x-y) d y, \quad 0<\alpha<n .
$$

We can write

$$
I_{\alpha, \Omega} f(x)=\frac{1}{2} \int_{\Sigma^{n-1}} \Omega(x, \theta) I_{\alpha, \theta} f(x) d \theta,
$$

where $I_{\alpha, \theta} f(x)=\int_{\mathbb{R}} f(x-t \theta)|t|^{\alpha-1} d t$ is the directional Riesz potential. The boundedness of $I_{\alpha, \Omega}$ from $L^{p}\left(\mathbb{R}^{n}\right)$ to $L^{q}\left(\mathbb{R}^{n}\right)$ is closely related to $(1.1)$ for $S_{\theta}=I_{\alpha, \theta}$; see $[10,11]$. The study of some types of maximal functions and maximal singular integrals rely on (1.1) for $S_{\theta}=M_{\theta}$, where

$$
M_{\theta} f(x)=\sup _{h>0} \frac{1}{h} \int_{0}^{h}|f(x-t \theta)| d t ;
$$

see $[6,7,10-12]$. Several similar results can also be found in $[1,4,5,8,9]$, and the references therein.

In this paper we extend $M_{\theta}$ to a more general form $\mathcal{M}_{\phi, \theta}^{-}$and investigate the mixed norm inequality

$$
\left(\int_{\mathbb{R}^{n}}\left(\int_{\Sigma^{n-1}} \Omega(x, \theta) \mathcal{M}_{\phi, \theta}^{-} f(x)^{r} d \theta\right)^{q / r} d x\right)^{1 / q} \leq C_{\Omega}\left(\int_{\mathbb{R}^{n}}|f(x)|^{p} d x\right)^{1 / p}
$$

for $1 \leq r \leq q \leq p<\infty$, where $\Omega$ is a nonnegative function on $\mathbb{R}^{n} \times \Sigma^{n-1}$. The directional maximal operator $\mathcal{M}_{\phi, \theta}^{-}$is defined as follows. Let $\phi$ be a nonnegative measurable function defined on $D=\left\{(z, t) \in \mathbb{R}^{2}: t<z\right\}$. For any measurable function $f$ on $\mathbb{R}$, we define the one-sided maximal function as

$$
M_{\phi}^{-} f(z):=\sup _{s<z} \frac{1}{\int_{s}^{z} \phi(z, t) d t} \int_{s}^{z} \phi(z, t)|f(t)| d t, \quad z \in \mathbb{R} .
$$

Let $\theta \in \Sigma^{n-1}, L_{\theta}=\{a \theta: a \in \mathbb{R}\}$, and let $L_{\theta}^{\perp}$ be the orthogonal complement of $L_{\theta}$ in $\mathbb{R}^{n}$. For any $x \in \mathbb{R}^{n}$, there exists a unique $x_{1} \in \mathbb{R}$ and $\bar{x} \in L_{\theta}^{\perp}$ such that $x=x_{1} \theta+\bar{x}$. For any measurable function $f$ on $\mathbb{R}^{n}$, we define the directional maximal function $\mathcal{M}_{\phi, \theta}^{-} f$ from $M_{\phi}^{-} f$ by

$$
\mathcal{M}_{\phi, \theta}^{-} f(x):=\sup _{h>0} \frac{1}{\int_{0}^{h} \phi\left(x_{1}, x_{1}-y\right) d y} \int_{0}^{h} \phi\left(x_{1}, x_{1}-y\right)|f(x-y \theta)| d y, \quad x \in \mathbb{R}^{n} .
$$


If $\phi \equiv 1$, then $\mathcal{M}_{\phi, \theta}^{-}=M_{\theta}$. Using Hölder's inequality, we see that the boundedness of operators of the form

$$
\mathbb{T}_{\Omega} f(x)=\int_{\Sigma^{n-1}} \Omega(x, \theta) \mathcal{M}_{\phi, \theta}^{-} f(x) d \theta
$$

from $L^{p}\left(\mathbb{R}^{n}\right)$ to $L^{q}\left(\mathbb{R}^{n}\right)$ is closely related to (1.2). The purpose of this paper is to establish (1.2) for $1 \leq r \leq q \leq p<\infty$. Based on the idea given in [9, Section 4.3], we show that (1.2) can be obtained by norm inequalities for $M_{\phi}^{-}$. In general, we consider the weighted inequality

$$
\left(\int_{\mathbb{R}} M_{\phi}^{-} f(z)^{q} u(z) d z\right)^{1 / q} \leq C_{\phi}\left(\int_{\mathbb{R}}|f(z)|^{p} v(z) d z\right)^{1 / p},
$$

where $1<p, q<\infty, u$ and $v$ are weights, and the constant $C_{\phi}$ is independent of $f$. Here a weight is a locally integrable function which is positive almost everywhere on $\mathbb{R}$. We prove (1.4) under some increasing conditions on $\phi$ and also give the estimates of $C_{\phi}$. Then the particular case $p=q$ and $u=v \equiv 1$ of (1.4) is applied to obtain (1.2). We also establish the estimates of $C_{\Omega}$.

Throughout this paper, we assume that all functions are measurable on their domains. For $0<z<\infty$, we define $z^{*}$ by $1 / z+1 / z^{*}=1$. We also take $0^{0}=\infty^{0}=1$ and $\infty / \infty=0 / 0=0 \cdot \infty=0$.

\section{Main results}

We first show that (1.2) can be obtained by (1.4). The method of the proof is based on the idea given in [9, Section 4.3].

THeorem 2.1. Let $1 \leq r \leq q \leq p<\infty$. Let $\Omega$ be a nonnegative function on $\mathbb{R}^{n} \times \Sigma^{n-1}$. Suppose that there exist $1 \leq \beta \leq q / r, 0 \leq m \leq \beta^{*}$, and a positive function $w$ on $\Sigma^{n-1}$ such that

$$
U(\beta, m):=\left(\int_{\Sigma^{n-1}}\left(\int_{\mathbb{R}^{n}} A(x, \theta)^{p /(p-q)} d x\right)^{r \beta(p-q) / p q} w(\theta)^{1-\beta} d \theta\right)^{1 / r \beta}<\infty,
$$

where

$$
A(x, \theta)=\Omega(x, \theta)^{\left(1-m / \beta^{*}\right) q / r}\left(\int_{\Sigma^{n-1}} \Omega(x, \tau)^{m} w(\tau) d \tau\right)^{q / r \beta^{*}} .
$$

If (1.4) holds for $p=q$ and $u=v \equiv 1$ with constant $C_{\phi}$, then we obtain (1.2) with

$$
C_{\Omega} \leq C_{\phi} U(\beta, m) .
$$

Proof of Theorem 2.1. Since

$$
\begin{aligned}
\int_{\Sigma^{n-1}} & \Omega(x, \theta) \mathcal{M}_{\phi, \theta}^{-} f(x)^{r} d \theta \\
& =\int_{\Sigma^{n-1}} \Omega(x, \theta)^{1-m / \beta^{*}+m / \beta^{*}} \mathcal{M}_{\phi, \theta} f(x)^{r} w(\theta)^{1 / \beta^{*}-1 / \beta^{*}} d \theta \\
& \leq \omega_{m}(x)^{1 / \beta^{*}}\left(\int_{\Sigma^{n-1}} \Omega(x, \theta)^{\left(1-m / \beta^{*}\right) \beta} \mathcal{M}_{\phi, \theta}^{-} f(x)^{\beta r} w(\theta)^{1-\beta} d \theta\right)^{1 / \beta},
\end{aligned}
$$


where $\omega_{m}(x)=\int_{\Sigma^{n-1}} \Omega(x, \tau)^{m} w(\tau) d \tau$, by Minkowski’s integral inequality

$$
\begin{aligned}
\left(\int_{\mathbb{R}^{n}}\right. & \left.\left(\int_{\Sigma^{n-1}} \Omega(x, \theta) \mathcal{M}_{\phi, \theta}^{-} f(x)^{r} d \theta\right)^{q / r} d x\right)^{1 / q} \\
& \leq\left(\int_{\mathbb{R}^{n}}\left(\int_{\Sigma^{n-1}} \Omega(x, \theta)^{\left(1-m / \beta^{*}\right) \beta} \mathcal{M}_{\phi, \theta}^{-} f(x)^{\beta r} w(\theta)^{1-\beta} d \theta\right)^{q / r \beta} \omega_{m}(x)^{q / r \beta^{*}} d x\right)^{1 / q} \\
& \leq\left(\int_{\Sigma^{n-1}}\left(\int_{\mathbb{R}^{n}} \mathcal{M}_{\phi, \theta}^{-} f(x)^{q} A(x, \theta) d x\right)^{r \beta / q} w(\theta)^{1-\beta} d \theta\right)^{1 / r \beta}
\end{aligned}
$$

where $A(x, \theta)=\Omega(x, \theta)^{\left(1-m / \beta^{*}\right) q / r} \omega_{m}(x)^{q / r \beta^{*}}$. We have

$$
\int_{\mathbb{R}^{n}} \mathcal{M}_{\phi, \theta}^{-} f(x)^{q} A(x, \theta) d x \leq\left(\int_{\mathbb{R}^{n}} \mathcal{M}_{\phi, \theta}^{-} f(x)^{p} d x\right)^{q / p}\left(\int_{\mathbb{R}^{n}} A(x, \theta)^{p /(p-q)} d x\right)^{(p-q) / p} .
$$

If (1.4) holds for $p=q$ and $u=v \equiv 1$ with constant $C_{\phi}$, then

$$
\begin{aligned}
\int_{\mathbb{R}^{n}} \mathcal{M}_{\phi, \theta}^{-} f(x)^{p} d x & =\int_{L_{\theta}^{\perp}} \int_{\mathbb{R}} \mathcal{M}_{\phi, \theta}^{-} f\left(x_{1} \theta+\bar{x}\right)^{p} d x_{1} d \bar{x} \\
& =\int_{L_{\theta}^{\perp}} \int_{\mathbb{R}} M_{\phi}^{-}(f(\cdot \theta+\bar{x}))\left(x_{1}\right)^{p} d x_{1} d \bar{x} \\
& \leq C_{\phi}^{p} \int_{L_{\theta}^{\perp}} \int_{\mathbb{R}}\left|f\left(x_{1} \theta+\bar{x}\right)\right|^{p} d x_{1} d \bar{x}=C_{\phi}^{p} \int_{\mathbb{R}^{n}}|f(x)|^{p} d x .
\end{aligned}
$$

Putting (2.3)-(2.5) together yields (1.2) and (2.2).

In the case $p=q$, the item $\left(\int_{\mathbb{R}^{n}} A(x, \theta)^{p /(p-q)} d x\right)^{r \beta(p-q) / p q}$ in (2.1) is understood to be ( $\left.\sup _{x \in \mathbb{R}^{n}} A(x, \theta)\right)^{r \beta / q}$. If $\beta=1$, then (2.1) can be reduced to

$$
U(1, m)=\left(\int_{\Sigma^{n-1}}\left(\int_{\mathbb{R}^{n}} \Omega(x, \theta)^{p q /(p r-q r)} d x\right)^{r(p-q) / p q} d \theta\right)^{1 / r}<\infty .
$$

If $\beta=q / r$, then $0 \leq m \leq(q / r)^{*}$ and (2.1) can be reduced to

$$
U(q / r, m)=\left(\int_{\Sigma^{n-1}}\left(\int_{\mathbb{R}^{n}} A(x, \theta)^{p /(p-q)} d x\right)^{(p-q) / p} w(\theta)^{1-q / r} d \theta\right)^{1 / q}<\infty,
$$

where

$$
A(x, \theta)=\Omega(x, \theta)^{m+(1-m) q / r}\left(\int_{\Sigma^{n-1}} \Omega(x, \tau)^{m} w(\tau) d \tau\right)^{q / r-1} .
$$

On the other hand, if $\Omega$ is independent of $x$, then we simply write $\Omega(x, \theta)=\Omega(\theta)$. In the case $p=q, U(\beta, m)$ in $(2.1)$ is reduced to

$$
U(\beta, m)=\left(\int_{\Sigma^{n-1}} \Omega(\theta)^{m} w(\theta) d \theta\right)^{1 / r \beta^{*}}\left(\int_{\Sigma^{n-1}} \Omega(\theta)^{\left(1-m / \beta^{*}\right) \beta} w(\theta)^{1-\beta} d \theta\right)^{1 / r \beta} .
$$


If we choose $\beta=p / r$, then there are three particular cases:

$$
\left\{\begin{array}{l}
U(p / r, 0)=\left(\int_{\Sigma^{n-1}} w(\theta) d \theta\right)^{1 / r-1 / p}\left(\int_{\Sigma^{n-1}} \Omega(\theta)^{p / r} w(\theta)^{1-p / r} d \theta\right)^{1 / p} \\
U\left(p / r,(p / r)^{*}\right)=\left(\int_{\Sigma^{n-1}} \Omega(\theta)^{p /(p-r)} w(\theta) d \theta\right)^{1 / r-1 / p}\left(\int_{\Sigma^{n-1}} w(\theta)^{1-p / r} d \theta\right)^{1 / p} \\
U(p / r, 1)=\left(\int_{\Sigma^{n-1}} \Omega(\theta) w(\theta) d \theta\right)^{1 / r-1 / p}\left(\int_{\Sigma^{n-1}} \Omega(\theta) w(\theta)^{1-p / r} d \theta\right)^{1 / p} .
\end{array}\right.
$$

The following theorem can be proved by a similar proof to that given in [13, Lemma 21.75 and Theorem 21.76].

Theorem 2.2. Let $1<p<\infty$. Suppose that $\sigma$ is a locally integrable function which is positive almost everywhere on $\mathbb{R}$. Let $M_{\sigma}^{-}$be defined as in $(1.3)$ with $\phi(z, t)$ replaced by $\sigma(t)$. Then for any nonnegative $f$ on $\mathbb{R}$,

$$
\left(\int_{\mathbb{R}} M_{\sigma}^{-} f(z)^{p} \sigma(z) d z\right)^{1 / p} \leq p^{*}\left(\int_{\mathbb{R}}|f(z)|^{p} \sigma(z) d z\right)^{1 / p} .
$$

In the following we establish (1.4) under some increasing conditions on $\phi$. Suppose that $1<p, q<\infty, u$ and $v$ are weights, and $\sigma=g^{p^{*}} v^{1-p^{*}}$. Then for $0 \leq \epsilon \leq \min \{1, p / q\}$ we define

$$
\begin{aligned}
U_{\phi}^{\epsilon}(z) & =\sup _{s<z} \frac{1}{\int_{s}^{z} \phi(z, t) d t} \int_{s}^{z} \phi(z, t) \frac{\sigma}{g}(t)\left(\int_{t}^{z} \sigma(y) d y\right)^{(\epsilon-1) / p} d t, \\
U_{\phi}^{\epsilon} & =\left(\int_{\mathbb{R}} U_{\phi}^{\epsilon}(z)^{p q /(p-\epsilon q)} u(z)^{p /(p-\epsilon q)} \sigma(z)^{\epsilon q /(\epsilon q-p)} d z\right)^{(p-\epsilon q) / p q} .
\end{aligned}
$$

In the case $p \leq q$ and $\epsilon=p / q$, it is understood that

$$
U_{\phi}^{\epsilon}=\sup _{z \in \mathbb{R}} U_{\phi}^{\epsilon}(z)(u(z) / \sigma(z))^{1 / q} .
$$

Theorem 2.3. Let $1<p, q<\infty$. Suppose that $\phi=g \psi$, where $g$ is a function positive almost everywhere on $\mathbb{R}, \psi$ is a nonnegative function defined on $D$, and $\psi(z, \cdot)$ is increasing and left continuous for each $z \in \mathbb{R}$. Suppose that $u$ and $v$ are weights such that $\sigma=g^{p^{*}} v^{1-p^{*}}$ is locally integrable. Then (1.4) holds with

$$
C_{\phi} \leq \inf _{0 \leq \epsilon \leq \min \{1, p / q\}}\left(\frac{1}{p^{*}}+\frac{\epsilon}{p}\right)\left(p^{*}\right)^{\epsilon} U_{\phi}^{\epsilon}
$$

Proof. It suffices to prove (1.4) for nonnegative $f$. Let $a<z$. Let $\Lambda_{\psi(z,)}$ be the Lebesgue-Stieltjes measure on $(-\infty, z)$ generated by $\psi(z, \cdot)$ defined by $\Lambda_{\psi(z, \cdot)}([a, b))=$ $\psi(z, b)-\psi(z, a)$ for $[a, b) \subset(-\infty, z)$. Then $\psi(z, t)=\psi(z, a)+\int_{[a, t)} d \Lambda_{\psi(z, \cdot)}$ for all $a<$ $t<z$. Let $h=(g / v)^{1-p^{*}} f$. By Fubini's theorem we see that, for any nonnegative $f$,

$$
\int_{a}^{z} \phi(z, t) f(t) d t=\psi(z, a) \int_{a}^{z} \sigma(t) h(t) d t+\int_{[a, z)}\left(\int_{s}^{z} \sigma(t) h(t) d t\right) d \Lambda_{\psi(z, \cdot)} .
$$


Let $M_{\sigma}^{-}$be defined as in (1.3) with $\phi(z, t)$ replaced by $\sigma(t)$. Then

$$
\int_{s}^{z} \sigma(t) h(t) d t \leq\left(\int_{s}^{z} \sigma(t) d t\right) M_{\sigma}^{-} h(z)
$$

for $a \leq s<z$. On the other hand, by Hölder's inequality,

$$
\int_{s}^{z} \sigma(t) h(t) d t \leq\left(\int_{s}^{z} \sigma(t) d t\right)^{1 / p^{*}}\left(\int_{\mathbb{R}} h(t)^{p} \sigma(t) d t\right)^{1 / p} .
$$

Therefore, for any $0 \leq \epsilon \leq \min \{1, p / q\}$,

$$
\int_{s}^{z} \sigma(t) h(t) d t \leq\left(\int_{s}^{z} \sigma(t) d t\right)^{1 / p^{*}+\epsilon / p} M_{\sigma}^{-} h(z)^{\epsilon}\left(\int_{\mathbb{R}} h(t)^{p} \sigma(t) d t\right)^{(1-\epsilon) / p}
$$

for $a \leq s<z$. By (2.8)-(2.9),

$$
\int_{a}^{z} \phi(z, t) f(t) d t \leq \Psi(z) M_{\sigma}^{-} h(z)^{\epsilon}\left(\int_{\mathbb{R}} h(t)^{p} \sigma(t) d t\right)^{(1-\epsilon) / p},
$$

where

$$
\Psi(z)=\psi(z, a)\left(\int_{a}^{z} \sigma(t) d t\right)^{1 / p^{*}+\epsilon / p}+\int_{[a, z)}\left(\int_{s}^{z} \sigma(t) d t\right)^{1 / p^{*}+\epsilon / p} d \Lambda_{\psi(z,)} .
$$

If $\psi(z, \cdot)$ is bounded on $(-\infty, z)$, then, using integration by parts,

$$
\Psi(z)=\left(\frac{1}{p^{*}}+\frac{\epsilon}{p}\right) \int_{a}^{z} \psi(z, s) \sigma(s)\left(\int_{s}^{z} \sigma(t) d t\right)^{(\epsilon-1) / p} d s .
$$

This implies that

$$
\begin{aligned}
\int_{a}^{z} \phi(z, t) f(t) d t \leq\left(\frac{1}{p^{*}}\right. & \left.+\frac{\epsilon}{p}\right) M_{\sigma}^{-} h(z)^{\epsilon}\left(\int_{\mathbb{R}} h(t)^{p} \sigma(t) d t\right)^{(1-\epsilon) / p} \\
& \times \int_{a}^{z} \psi(z, s) \sigma(s)\left(\int_{s}^{z} \sigma(t) d t\right)^{(\epsilon-1) / p} d s .
\end{aligned}
$$

This inequality still holds when $\psi(z, \cdot)$ is not bounded on $(-\infty, z)$ since we can replace $\psi$ by $\psi_{m}$ in $(2.10)$, where $\left\{\psi_{m}(z, \cdot)\right\}$ is an increasing sequence of bounded increasing and left continuous functions such that $\psi_{m}(z, \cdot) \rightarrow \psi(z, \cdot)$ as $m \rightarrow \infty$, and then by letting $m \rightarrow \infty$ and applying the monotone convergence theorem. Therefore

$$
M_{\phi}^{-} f(z) \leq\left(\frac{1}{p^{*}}+\frac{\epsilon}{p}\right) U_{\phi}^{\epsilon}(z) M_{\sigma}^{-} h(z)^{\epsilon}\left(\int_{\mathbb{R}} h(t)^{p} \sigma(t) d t\right)^{(1-\epsilon) / p} .
$$

Now

$$
\begin{aligned}
& \left(\int_{\mathbb{R}} M_{\phi}^{-} f(z)^{q} u(z) d z\right)^{1 / q} \\
& \quad \leq\left(\frac{1}{p^{*}}+\frac{\epsilon}{p}\right)\left(\int_{\mathbb{R}} M_{\sigma}^{-} h(z)^{q \epsilon} U_{\phi}^{\epsilon}(z)^{q} u(z) d z\right)^{1 / q}\left(\int_{\mathbb{R}} h(z)^{p} \sigma(z) d z\right)^{(1-\epsilon) / p} .
\end{aligned}
$$


By Hölder's inequality,

$$
\begin{aligned}
\int_{\mathbb{R}} M_{\sigma}^{-} h(z)^{q \epsilon} U_{\phi}^{\epsilon}(z)^{q} u(z) d z & \leq\left(U_{\phi}^{\epsilon}\right)^{q}\left(\int_{\mathbb{R}} M_{\sigma}^{-} h(z)^{p} \sigma(z) d z\right)^{q \epsilon / p} \\
& \leq\left(p^{*}\right)^{q \epsilon}\left(U_{\phi}^{\epsilon}\right)^{q}\left(\int_{\mathbb{R}} h(z)^{p} \sigma(z) d z\right)^{q \epsilon / p} .
\end{aligned}
$$

This implies (1.4) with $C_{\phi} \leq\left(1 / p^{*}+\epsilon / p\right)\left(p^{*}\right)^{\epsilon} U_{\phi}^{\epsilon}$. This estimate holds for arbitrary $0 \leq \epsilon \leq \min \{1, p / q\}$ and therefore (2.7) is obtained.

If we choose $\epsilon=0$, then (2.7) can be reduced to

$$
C_{\phi} \leq \frac{1}{p^{*}}\left(\int_{\mathbb{R}} U_{\phi}^{0}(z)^{q} u(z) d z\right)^{1 / q},
$$

where $U_{\phi}^{0}(z)$ is given in (2.6) with $\epsilon=0$. In the case $q<p$, if we choose $\epsilon=1$, then $U_{\phi}^{1}(z)=M_{\phi}^{-}(\sigma / g)(z)$ and (2.7) can be reduced to

$$
C_{\phi} \leq p^{*}\left(\int_{\mathbb{R}} M_{\phi}^{-}(\sigma / g)(z)^{p q /(p-q)} u(z)^{p /(p-q)} \sigma(z)^{q /(q-p)} d z\right)^{(p-q) / p q} .
$$

In the case $p \leq q$, if we choose $\epsilon=p / q$, then the estimate in (2.7) is reduced to

$$
C_{\phi} \leq\left(\frac{1}{p^{*}}+\frac{1}{q}\right)\left(p^{*}\right)^{p / q} \sup _{z \in \mathbb{R}} U_{\phi}^{p / q}(z)\left(\frac{u(z)}{\sigma(z)}\right)^{1 / q},
$$

where $U_{\phi}^{p / q}(z)$ is given in (2.6) with $\epsilon=p / q$. In particular, if $p=q$ and $u=v$, then (2.11) is reduced to

$$
C_{\phi} \leq p^{*} \sup _{z \in \mathbb{R}} M_{\phi}^{-}(\sigma / g)(z)(g(z) / \sigma(z)) .
$$

If $g / v$ is increasing, then $M_{\phi}^{-}(\sigma / g)(z) \leq \sigma(z) / g(z)$ and so $C_{\phi} \leq p^{*}$.

Corollary 2.4. Let $1<p<\infty$. Suppose that $\phi=g \psi$, where $g$ is a locally integrable function which is positive almost everywhere on $\mathbb{R}, \psi$ is a nonnegative function defined on $D$, and $\psi(z, \cdot)$ is increasing and left continuous for each $z \in \mathbb{R}$. Suppose that $v$ is a weight such that $g / v$ is increasing. Then

$$
\left(\int_{\mathbb{R}} M_{\phi}^{-} f(z)^{p} v(z) d z\right)^{1 / p} \leq p^{*}\left(\int_{\mathbb{R}}|f(z)|^{p} v(z) d z\right)^{1 / p} .
$$

The following corollary can be obtained by Theorem 2.1 and Corollary 2.4 with $v \equiv 1$.

Corollary 2.5. Let $1 \leq r \leq q \leq p<\infty$. Let $\Omega, \beta, m$, and $w$ be given as in Theorem 2.1. Suppose that $\phi=g \psi$, where $g$ and $\psi$ are as given in Corollary 2.4, and $g$ is increasing. Then (1.2) holds with

$$
C_{\Omega} \leq p^{*} U(\beta, m)
$$

where $U(\beta, m)$ is defined in (2.1). 


\section{References}

[1] N. Bez, 'Mixed-norm estimates for a class of nonisotropic directional maximal operators and Hilbert transforms', J. Funct. Anal. 255 (2008), 3281-3302.

[2] A. P. Calderón and A. Zygmund, 'On singular integrals', Amer. J. Math. 78 (1956), 289-309.

[3] A. P. Calderón and A. Zygmund, 'On singular integrals with variable kernel', Appl. Anal. 7 (1978), 221-238.

[4] L.-K. Chen, 'The singular integrals related to the Calderón-Zygmund method of rotations', Appl. Anal. 30 (1988), 319-329.

[5] L.-K. Chen, 'The maximal operators related to the Calderón-Zygmund method of rotations', Illinois J. Math. 33 (1989), 268-279.

[6] M. Christ, J. Duoandikoetxea and J. L. Rubio de Francia, 'Maximal operators related to the Radon transform and the Calderón-Zygmund method of rotations', Duke Math. J. 53 (1986), 189-209.

[7] M. Cowling and G. Mauceri, 'Inequalities for some maximal functions, I', Trans. Amer. Math. Soc. 287 (1985), 431-455.

[8] J. Duoandikoetxea, 'Weighted norm inequalities for homogeneous singular integrals', Trans. Amer. Math. Soc. 336 (1993), 869-880.

[9] J. Duoandikoetxea, Fourier Analysis, Graduate Student in Mathematics, 29 (American Mathematical Society, Providence, RI, 2001).

[10] J. Duoandikoetxea, 'Directional operators and mixed norms', Publ. Mat. Special Issue (2002), 3956 (Proceedings of the 6th International Conference on Harmonic Analysis and Partial Differential Equations).

[11] J. Duoandikoetxea and O. Oruetxebarria, 'Mixed norm inequalities for directional operators associated to potentials', Potential Anal. 15 (2001), 273-283.

[12] R. Fefferman, 'On an operator arising in the Calderón-Zygmund method of rotations and the Bramble-Hilbert lemma', Proc. Natl. Acad. Sci. USA 80 (1983), 3877-3878.

[13] E. Hewitt and K. Stromberg, Real and Abstract Analysis (Springer, Berlin, 1965).

DAH-CHIN LUOR, Department of Applied Mathematics, I-Shou University, Dashu District, Kaohsiung City 84001, Taiwan

e-mail: dclour@isu.edu.tw 